\title{
E-Health for Individualized Prevention of Eating Disorders
}

\author{
Katajun Lindenberg ${ }^{1,}$, Markus Moessner ${ }^{1}$, Joanna Harney ${ }^{2}$, Orla McLaughlin ${ }^{2}$ and Stephanie Bauer ${ }^{1}$ \\ ${ }^{1}$ University Hospital Heidelberg, Center for Psychotherapy Research, Heidelberg, Germany \\ ${ }^{2}$ Trinity College Dublin, Student Counseling Service, Dublin, Ireland
}

\begin{abstract}
In the field of illness prevention, it is becoming increasingly important that effective treatments be broadly disseminated and easily accessible to large populations located over wide geographical areas. The internet offers many opportunities to improve illness prevention and has become an important tool for both providers and users. An increasing number of users are looking for help on web-pages, in forums and chat rooms, to access information and exchange experiences with other users or counselors. Appetite for Life is an Internet-based program for the prevention of eating disorders (ED) in college students. It provides individualized support to students at-risk of developing an ED. Depending on initial screening results, specific program modules are recommended to the user, matching their individual needs. The program contains a web-page with psychoeducational information on ED, an anonymous forum to receive and provide peer support, a supportive monitoring and feedback program and a chat platform, which can be used to communicate with professional counselors and other users. If needed, users can be referred to face-to-face counseling.

In this paper we report experience in practical use of the program at the Trinity College Dublin. Participants could anonymously register and individually choose the length of participation in the prevention program. Two months after registration and at the time of deregistration, participants were asked to evaluate the program. Data of $\mathrm{N}_{\text {screened }}=457$ and

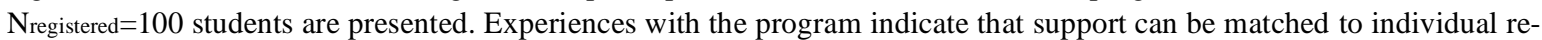
quirements by providing Internet-delivered stepped-care modules that encourage the user to seek support according to their personal preferences and needs.
\end{abstract}

Keywords: Eating disorders, E-health, prevention.

\section{INTRODUCTION}

Eating disorders (ED) are associated with enormous psychological distress, social and interpersonal impairment and reduced quality of life. Typically, affected individuals become ill at an early age and many are at substantial risk of developing a chronic course of illness, involving recurrent intensive treatments [1,2]. Even the best available treatments for ED fail to help a large number of patients and response rates have stayed disappointingly low in adult samples over the years [3-5].

Many affected individuals seek professional help after suffering for many years. This is partly due to shame and stigma around ED [6] and to a lack of easy-access specialized care. Since longer illness duration is associated with poorer prognosis and risk for a chronic course of illness, it is crucial to provide support at an early stage, ideally before the manifestation of a full-blown ED [7]. Thus, broad availability and accessibility of ED prevention programs are of particular importance.

However, ED prevention and early intervention efforts are hampered by the fact that the development of ED follow a heterogeneous and largely unknown course. Although a variety of risk factors have been discussed in the literature [8], little is known about their temporal relationship and their

*Address correspondence to this author at the Center For Psychotherapy Research, Bergheimer Str. 54, 69115 Heidelberg, Germany; Tel: +49 6221 / 568192; Fax: +49 6221 / 567350; E-mail: katajun.lindenberg@med.uniheidelberg.de, lindenberg@psyres.de differential contribution to the actual onset of an ED. As of today, it can neither be predicted reliably which individuals will develop an ED nor at which point in time the onset will most likely occur. However, some factors have been found to be associated with an increased risk for the development of eating disordered attitudes and behaviors, e.g. excessive weight and shape concerns, dietary restraint [8]. The assessment of these factors in large samples allows for the identification of high risk subgroups in which these factors can then be targeted via selective prevention efforts.

As in other areas of mental health care, programs based on information and communication technology (ICT) are increasingly used to enhance preventive efforts. ICTenhanced interventions offer several advantages for the field of prevention and allow treatments to overcome some of the limitations of traditional programs, especially with respect to their implementation and dissemination [9]: They allow interventions to be available to large populations (scalability) independent of time and place (accessibility), they ensure that specific contents of the intervention are delivered in the intended way without deviations over time (fidelity), and they can be maintained at lower cost than most traditional programs. The latter increases their chances of becoming part of routine health services beyond study settings (sustainability). Several ICT-enhanced programs for ED prevention have been introduced. Promising results have been found for the Internet-based cognitive-behavioral program 'Student Bodies', which led to a substantial reduction in weight and shape concerns in college-age women at-risk for the development of an ED [10]. 
However, despite these encouraging findings, a systematic review found no overall effect of Internet-based prevention programs on ED symptoms and called for refined approaches [11].

In light of the above mentioned heterogeneity in the development of ED, one limitation of existing programs seems to be their rigidity: Typically, they follow manualized approaches and require that all participants use the same amount and intensity of support for a pre-defined timelimited duration. The use of ICT offers promising opportunities to develop prevention programs that are more flexible than existing interventions in various respects: First, risk factors and ED-related attitudes and behaviors can be monitored online continuously over time, allowing for the timely identification of increases in impairment and manifestation of ED symptoms.

Second, support can be offered to participants flexibly depending on their needs, i.e. individuals with little impairment can engage in low-intensity modules whereas those with more impairment can engage in higher intensity treatment components. The availability of modules of various intensities corresponds to the idea of stepped care [12] and allows for the provision of individually tailored support [13]. Third, participants can receive support anonymously which may help to reduce psychological barriers to seeking help. The anonymous contact may then be used to facilitate access to regular health care for individuals with manifest ED symptoms for whom the online support appears to be insufficient.

Based on these considerations, the Internet-based program Es[s]prit was developed for the prevention and early intervention of ED [14]. Since symptoms of sub-clinical disordered eating as well as full-syndrome ED have been commonly found among college-age women, Es[s]prit was developed for this age group and offered in cooperation with the Student Counseling Center of the University of Heidelberg, Germany.

In accordance with the findings of Stice and Shaw's [15] meta-analytic review on ED prevention, Es[s]prit was designed for the prevention of ED, targeting populations that were shown to be at-risk for the development of an ED on the basis of an initial online screening (selective or targeted prevention). It also targeted individuals who reported early ED symptoms in the screening. Furthermore Es[s]prit comprises several interactive components which have been found to enhance the effectiveness of prevention programs [15]. These include an online forum that allows for peer support among participants and chat sessions that offer participants professional support from an online counselor. The components of the program are described in more detail below.

Following the completion of a pilot study in a German sample [14], Es[s]prit was translated into several languages including Spanish, French, Portuguese, and English. In this paper we report on the implementation of the English version of the program (entitled "Appetite for Life") in an Irish University setting. This study evaluates the implementation of the Appetite For Life program and its delivery to the target population, i.e., students enrolled at the Trinity College Dublin (TCD). Specifically, the success of the implementation will be evaluated by determining a) the proportion of the target population that completed the initial screening, and the proportion of the target population that registered for the program; b) the proportion of the population that used the various modules, c) the acceptability and user satisfaction rates and d) differences in the use of modules and acceptability between different risk groups.

\section{MATERIALS AND METHODOLOGY}

\section{"Appetite For Life" Modules}

Appetite For Life is a translation of the German program Es[s]prit [14]. Like the Es[s]prit, Appetite for Life contains five modules of increasing intensity.

\section{Screening}

The screening procedure is a prerequisite for registering on the program. It assesses the participants' current level of impairment and risk of developing an ED as a basis for recommendations for the use of specific Appetite For Life modules. Thus, the level of support can be adjusted according to participants' needs. The Weight Concern Scale (WCS) [16] serves to determine the participants' risk status, whereas the Short Evaluation of Eating Disorders (SEED) [17] assesses the occurrence of ED symptoms. The measures are explained below.

Screened participants were classified into four different risk groups. The "no-risk" group was defined by a score lower than 40 on the WCS and the absence of ED symptoms. The "moderate risk" group was defined by a WCS score of 40 or higher but below 60 . The "mild symptoms" group was defined by the absence of risk according to the WCS (scores lower than 40), but mild ED symptoms according to the SEED (e.g. slightly underweight or a low frequency of binge episodes). The "high risk" group was characterized by scores on the WCS of 60 or higher.

Students classified either into the "moderate risk", "high risk" or "mild symptoms" group were eligible and invited to register for full participation in Appetite For Life, whereas "no-risk" individuals were invited to register for participation in the low-intensity modules only. Participants were excluded from participation if 1) they were not enrolled at the TCD, 2) they were undergoing current treatment for an ED, 3) they reported severe ED symptoms or 4) they indicated that the data they entered did not apply to themselves. Participants received comprehensive feedback explaining their screening result, and if impairment was considered too severe, they were excluded from the study and directly referred to the cooperating TCD Student Counseling Center.

At registration, participants are able to choose a username and a password. The registration process requires the acceptance of the user conditions, the provision of a valid TCD email address and the confirmation of the registration email. Confidentiality and data security are guaranteed by the Appetite For Life provider.

\section{Psychoeducation and Peer Support}

The Appetite For Life website contains comprehensive information about ED in general as well as information on causes, triggers and first signs of an ED. The different forms of ED are explained in detail using personalized examples. Moreover, the website provides information about how ED 
can be detected early and how self-help strategies concerning body image, figure and weight or nutrition and eating behavior can be applied to prevent ED onset. The information pages are adapted from the German Federal Center for Health Education [18].

The Appetite For Life website further provides a moderated forum, where registered users can post messages on different topics and initiate discussions about ED. The main idea of the forum is to encourage users, who find themselves in similar situations, to share their experiences, answer questions in an anonymous, low intensity way and to support each other. The forum is monitored and moderated by the Appetite For Life team members.

Pro-anorectic or aggressive statements are prohibited and deleted by the moderator.

\section{Supportive Monitoring and Feedback System}

The development of ED symptoms is in general highly heterogeneous, and the time when disturbed eating behavior shifts to eating pathology is largely unknown. Therefore, the core module of Appetite For Life consists of a supportive monitoring and feedback system, which aims at continuous monitoring of ED related symptoms, early detection of eating pathology and providing early minimal intervention by supportive feedback. Thus, participants' symptoms are tracked closely over time, in order to identify early on when ED symptoms deteriorate, and to support participants by providing feedback on the four dimensions a) body dissatisfaction, b) preoccupation with body weight and shape, c) balanced diet and nutrition, and d) binges and compensatory behavior. If the course of ED symptoms deteriorates, the message expresses concern and recommends strategies to counteract the emergence of ED behaviors, whereas healthy eating behavior and improvements are reinforced by positive feedback. Individuals, who develop symptoms over time, can be identified early by an automated alarm system and referred to the consultation chat (see below), where anonymous contact can be used to motivate users to seek more intensive treatment, i.e., inpatient or outpatient treatment.

A weekly email reminds participants to complete the monitoring questionnaire, which can be accessed either via a link posted by email or via the Appetite For Life webpage. Following the completion of the questionnaires, the feedback message is automatically generated according to a predefined feedback algorithm. The database includes more than 2000 feedback messages. Detailed information about the feedback algorithm can be found in Bauer et al. [14]. The questionnaires, reminder emails and feedback messages are organized by the data management software Web-Akquasi [19].

\section{Consultation Chat}

Seeking help is difficult for many affected individuals, both due to feelings of shame and stigma and to the lack of easy-access specialized care. Many individuals get help late or never seek treatment. Therefore, a chat module was developed for Appetite For Life, providing anonymous, low intensity counseling for users, which also serves as a first contact point to help motivate affected individuals to seek professional help and to support them in finding inpatient/ outpatient treatment. The consultation chat is guided by a clinician and offered in two different settings to all registered participants with full access to the program. Once per month, participants were invited to join the group chat meeting, which aims to share experiences and discuss ED related questions. Group chat appointments are announced on the Appetite For Life website. Further, 30 minute one-to-one chat appointments with the online counselor are offered several times per week. One-to-one chat appointments can be booked online by participants. Moreover, counselors can directly invite specific participants to join a one-to-one chat session, e.g., if participants report ED symptoms above a pre-defined cut-off in the weekly monitoring. In this case, an alert mail is automatically generated and sent to the online counselor, who in turn encourages the respective participant via email to use the anonymous 30-minute one-to-one consultation chat. The online counselor can access all relevant data stored in the database to retrieve detailed information about the participant's symptom patterns as a basis for this chat session. The one-to-one consultation chat serves to clarify uncertainties and queries and, if necessary, motivate participants to seek more intensive forms of support, e.g. face-to-face counseling.

\section{Face-to-Face Counseling}

Appetite for Life aims at the prevention of ED and thus targets individuals, who are at-risk of developing an ED, but do not meet full ED criteria. However, it is possible that participants may develop severe ED symptoms during their participation, and in this case it is crucial to offer timely support. Therefore, if participants required more intensive support during their participation in Appetite For Life, they were referred to the face-to-face counseling module at the cooperating TCD Student Counseling Center, and if necessary, they were further referred to inpatient or outpatient treatment. This ensured timely provision of ED treatment without delays, which is known to be associated with better treatment outcomes [20].

\section{Procedure}

Appetite For Life was offered to TCD students in cooperation with the TCD Student Counseling center. Several staff members were trained by the German Es[s]prit Team on how to use and manage the program, e.g. to coordinate the chat sessions and moderate the forum. Ethical approval to conduct the Appetite For Life study was obtained at the end of November 2008 by the School of Psychology Research Ethics Committee. Appetite For Life was advertised to all students of the TCD by mass emails and posters beginning on the 22nd of January 2009. Further, the TCD website contained links announcing the Appetite For Life program. There were 15,914 registered students in 2008/09.

\section{Measures}

The WCS developed by Killen et al. [16] was used to screen participants for their risk of developing an ED and is scored from 0 to 100 . The WCS is a widely used screening instrument, e.g. $[10,14]$, and consists of 5 items.

The SEED [17] was used to assess core ED symptoms both at registration and in the weekly monitoring. The SEED consists of 6 items and reports total severity indices (TSI) for Anorexia (AN) and Bulimia (BN). 
Symptoms defining the BNTsI refer to binge eating, compensatory behavior and overconcern with body weight and shape, whereas the ANTSI refers to underweight, fear of weight gain and distortion of body perception.

To assess the psychological and ED related impairment of Appetite For Life users at registration, we used the 1) Clinical and Research Inventory for Eating Disorders (CREAT) [21], which consists of 70 items and 12 subscales, reported on a 6-point likert scale, and 2) the Eating Disorder Examination Questionnaire (EDE-Q) [22], a 28-item instrument, which assesses eating pathology on the four subscales restraint, eating concern, weight concern and shape concern.

The weekly monitoring questionnaire measures cognitive and behavioral correlates of ED on four dimensions: 1) body dissatisfaction, 2) over concern with body weight and shape, 3 ) unbalanced nutrition and dieting, and 4) binge eating and compensatory behaviors, and served as the basis for the supportive feedback messages.

Appetite For Life allows participants to individually decide on the length of participation in the prevention program. Therefore, participants were asked to evaluate the program both eight weeks after registration and at the end of participation (deregistration) in Appetite For Life. The acceptability questionnaire contained questions on the use of the different modules, user satisfaction and their acceptance of the program. Furthermore, information on the utilization of the Appetite For Life modules were stored directly in the database, such as the number of forum viewings per user, the number of forum posts and the number of weeks of using the monitoring program.

\section{RESULTS}

\section{Screening}

Between February and September 2009, N=457 (2.87\% of all TCD students) completed the initial screening. Twenty five participants $(5.47 \%)$ were excluded because they indicated that the data they entered did not apply to themselves (they had made up the data just to see what the screening result would be), 7 participants were excluded because of current ED treatment $(1.53 \%)$, and 115 (25.16\%) were excluded and directly referred to the TCD Student Counseling Center, because they reported severe ED symptoms.

In total, 310 participants $(67.83 \%$ of all screened participants) were invited for full or partial participation in Appetite For Life.

Forty-nine out of $310(15.80 \%)$ were invited for partial participation because of "no-risk", out of which 8 (16.33\%) registered to use the forum. 2 of them (25\%) finally activated their account. Two hundred and sixty-one participants out of $310(84.19 \%)$ were invited for full participation because of "moderate risk" ( $\mathrm{N}=74 ; 23.87 \%)$, "high risk" $(\mathrm{N}=173$; $55.80 \%)$ or "mild symptoms" ( $\mathrm{N}=14 ; 4.52 \%)$. Twenty-five (33.78\%) participants registered from the "moderate risk" group of which 21 (84\%) activated their account, 66 (38.15\%) registered from the "high risk" group where 56 (84.85\%) activated their account, and one participant (7.14\%) registered from the "mild symptoms" group, who also activated his account.

In summary, 310 out of 457 (67.83\%) screened participants were eligible to use Appetite For Life. One hundred actually registered $(32.26 \%)$. Whereas $92(92 \%)$ were eligible to use all the components, only $78(84.78 \%)$ of them activated their accounts (see Fig. 1).

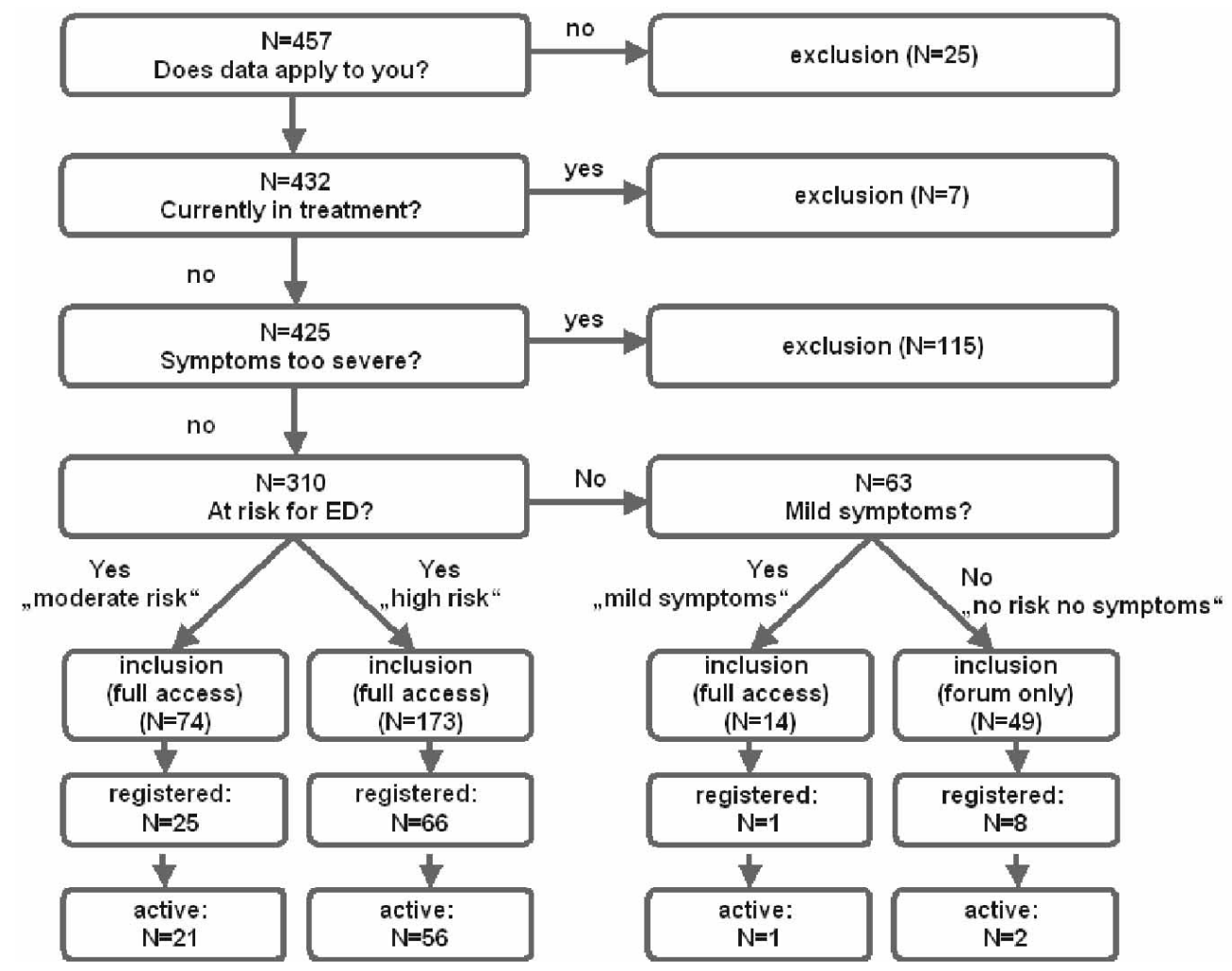

Fig. (1). Appetite For Life screening results. 
Note. The following data on the acceptability and user satisfaction of Appetite For Life refer to 8 out of 21 active "moderate risk" participants and 25 out of 56 active "high risk" participants, who answered the acceptability questionnaire. The "mild symptoms" group $(\mathrm{N}=1)$ was not analyzed separately, although this participant answered the questionnaire. Participants from the "no risk" group were not asked to evaluate the program, because they could use the forum and psychoeducation only.

\section{Description of the Sample}

Registered participants had a mean age of 23.11 ( $\mathrm{SD}=5.54)$, a mean $\mathrm{BMI}$ of 23.57 ( $\mathrm{SD}=4.81)$, and a mean WCS score of $66.16(\mathrm{SD}=19.39)$. Table 1 shows the screening data of all registered participants. On average, participants who registered to the program were more impaired than healthy norm samples in terms of their SEED, CR-EAT, and EDE-Q scores, but less impaired compared to clinical samples. Norm data for healthy and clinical samples can be found in $[17,21,23]$, respectively.

\section{Comparison of Registered vs. Non-Registered Students}

A one-way ANOVA was conducted to evaluate differences in terms of age, BMI, WCS, ANTSI, and BNTsi scores between eligible students, who did $(\mathrm{N}=100)$ versus who did not $(\mathrm{N}=210)$ register on the program. Registered participants were characterized by on average significantly higher scores on the WCS $(\mathrm{M}=66.16 \quad(\mathrm{SD}=19.39) \quad$ vs. $\mathrm{M}=57.94$ $(\mathrm{SD}=24.84), F(1,308)=8.48, p<.01)$, significantly higher BNTSi scores $(\mathrm{M}=1.01(\mathrm{SD}=0.58)$ vs. $\mathrm{M}=0.75(\mathrm{SD}=0.57), F$ $(1,308)=13.35, p<.01)$ and a significantly higher BMI $(\mathrm{M}=23.57 \quad(\mathrm{SD}=4.81)$ vs. $\mathrm{M}=22.49 \quad(\mathrm{SD}=4.06), \quad F \quad(1$, $308)=4.26, p=.040)$ compared to those eligible participants who did not register. However, registered participants did not differ significantly in age $(\mathrm{M}=23.11$ ( $\mathrm{SD}=5.54)$ vs. $\mathrm{M}=23.59 \quad(\mathrm{SD}=7.25), \quad F(1,308)=.34, \quad$ n.s. $)$ and ANTSI $(\mathrm{M}=0.81(\mathrm{SD}=0.31)$ vs. $\mathrm{M}=0.73(\mathrm{SD}=0.38), F(1,308)=3.22$, n.s.) from non-registered students.

\section{The Use of Different Appetite For Life Modules}

\section{Total Sample}

Seventy-eight out of $92(84.78 \%)$ participants, who were eligible to use all the components, activated their account by clicking the respective link in the registration email.

The use of the different Appetite for Life modules was assessed in two ways: information on the use of the monitoring and the forum could be assessed objectively by retrieving data from the database (data on 78 active users available), whereas information on the use of the information pages and the chat were retrieved from self-report data from the acceptability questionnaire (data on 34 active users available; 8 at "moderate risk", 25 at "high risk" and one from the "mild symptom" group).

Table 1. Psychological and ED Related Impairment of Registered Participants

\begin{tabular}{|c|c|c|c|}
\hline Measure & & Mean & $S D$ \\
\hline $\operatorname{SEED}(\mathrm{N}=100)$ & $\mathrm{AN}_{\mathrm{TSI}}$ & 0.81 & 0.31 \\
\hline \multirow[t]{4}{*}{ EDE-Q $(\mathrm{N}=43)$} & Restraint & 3.06 & 1.41 \\
\hline & Eating Concern & 2.85 & 1.63 \\
\hline & Shape Concern & 4.13 & 1.25 \\
\hline & EDE-Q total score & 3.43 & 1.17 \\
\hline \multirow[t]{8}{*}{ CR-EAT $(\mathrm{N}=43)$} & Weight Preoccupation & 4.40 & 0.92 \\
\hline & Self-esteem & 3.35 & 0.93 \\
\hline & Concerns about Negative Evaluation & 4.58 & 0.83 \\
\hline & Body Embarrassment & 4.13 & 1.08 \\
\hline & Restrained Eating Behaviour & 3.23 & 1.14 \\
\hline & Societal Expectations of Weight and Shape & 3.63 & 1.03 \\
\hline & Perfectionism: Familial Expectations & 3.02 & 1.33 \\
\hline & Harmful Weight Regulation & 3.03 & 1.34 \\
\hline
\end{tabular}


Thirteen (16.67\%) active users, didn't use the monitoring function, whereas $16(20.51 \%)$ used the monitoring for one week and $49(62.82 \%)$ participated in the monitoring program for at least two weeks. The average duration of active participants using the monitoring program was 4.36 weeks $(\mathrm{SD}=5.39)$. Thirty-six $(46.15 \%)$ active users accessed the forum posts, whereas only $8(10.26 \%)$ posted messages in the forum. According to self-report data assessed via the acceptability questionnaire, the information pages were used by 28 out of $34(82.35 \%)$ users and the chat by 2 out of 34 $(5.88 \%)$ users. However, only 34 out of $78(43.59 \%)$ active users answered the acceptability questionnaire; 4 (11.76\%) of these were at 2 month follow up and $30(88.24 \%)$ at the end of participation. Therefore a one-way ANOVA was conducted to test whether this sub-sample of participants, who completed the acceptability questionnaire showed systematic differences compared to those users, who did not complete it. Results showed that these participants did not differ in level of impairment, BMI, age, or WCS.

\section{Moderate Risk vs. High Risk Participants}

Following the recommendations of the program, "moderate risk" participants should have mainly used the low intensity modules, whereas "high risk" participants should have used the higher intensity modules more frequently. To assess whether the idea of matching support intensity to individuals' needs was reflected in the actual use of the program, the usage of the various Appetite For Life modules was analyzed separately for "moderate risk" vs. "high risk" participants. Due to the small sample size, the "mild symptoms" group was not analyzed separately. Data on the use of the information pages were assessed as self report data from the acceptability questionnaire (data available on $\mathrm{N}=8$ "moderate risk" and $\mathrm{N}=25$ "high risk" participants), whereas data on the use of the forum and the monitoring program were directly retrieved from the database (data available on $\mathrm{N}=21$ "moderate risk" and N=56 "high risk" participants). The use of the information pages was comparable for the "moderate risk" and "high risk" group participants: 7 out of $8(87.50 \%)$ vs. 20 out of $25(80 \%)$ used the information pages, whereas the forum and the monitoring were used more often by "high risk" participants. In the "moderate risk" group, 7 out of $21(33.33 \%)$ had accessed the forum posts and 1 out of 21 $(4.76 \%)$ had posted messages on the forum. However, in the "high risk" group, 29 out of $56(51.79 \%)$ had accessed the forum posts and 7 out of $56(12.50 \%)$ had posted at least one message. In the "moderate risk" group, 5 out of $21(23.81 \%)$ didn't use the monitoring, 6 out of $21(28.57 \%)$ had used the monitoring for one week only and 10 out of 21 (47.62\%) had used the monitoring for at least two weeks. In the "high risk" group, 7 out of $56(12.50 \%)$ had never used the monitoring, 10 out of $56(17.86 \%)$ had used the monitoring for one week and 39 out of $56(69.64 \%)$ had used the monitoring for at least two weeks.

\section{Acceptability and User Satisfaction}

\section{Total Sample}

The Appetite For Life acceptability questionnaire included questions about whether participants had ever dealt with the subject of ED before, how they found out about the program and why they registered to the program. More than half of all users (19 out of $34 ; 55.88 \%$ ) indicated that partici- pating in Appetite For Life was the first time, that they had dealt with ED. Twenty-three out of 34 participants (67.65\%) found out about the Appetite For Life program through Internet searches, $1(2.94 \%)$ by an e-mail from the University, $1(2.94 \%)$ through a friend, and $9(26.47 \%)$ by other means. When asked for the main reason for registering on the program, 16 out of $34(47.06 \%)$ indicated that they were curious about it, 12 (35.29\%) had been actually looking for help, 3 $(8.82 \%)$ had specific questions they wanted to be answered and $3(8.82 \%)$ named other reasons.

Moreover, participants were asked to evaluate the various components of Appetite For Life. They were allowed to answer "don't know", if they had not used a specific component. Therefore, the total number of ratings per component varied between 23 and 34 (see Table 2).

When participants were asked to evaluate the program in general, 7 out of $34(20.59 \%)$ indicated that they had gained knowledge on ED by participating in Appetite For Life, 5 $(14.71 \%)$ said that their participation in Appetite For Life had helped them to clarify certain questions, $3(8.82 \%)$ said that without Appetite For Life, they wouldn't have known who to share their problems with, and 12 (35.29\%) indicated that overall, they were pleased with Appetite For Life. Further, 13 out of $34(38.24 \%)$ said that they would use Appetite For Life again and $16(47.06 \%)$ would recommend Appetite For Life to a friend with ED problems.

When we asked the participants whether their participation in Appetite For Life was helpful, 15 out of 34 (44.12\%) said that it was 'helpful', $17(50 \%)$ said it was neither helpful nor harmful ('neutral'), and $2(5.88 \%)$ said it was 'harmful'.

\section{Moderate Risk vs. High Risk Participants}

The acceptability and user satisfaction of the program were analyzed for the "moderate risk" $(\mathrm{N}=8)$ and the "high risk" ( $\mathrm{N}=25)$ groups separately.

Overall, the concepts of the various components were rated more positively by the "moderate risk" group, whereas participation in Appetite For Life in general was rated more positively by "high risk" participants (see Table 3 ).

\section{DISCUSSION}

The purpose of this study was the evaluation of an individualized approach to ED prevention and the acceptability of the program in an Irish University setting. Because of the low incidence of ED in the population, only a small proportion is likely to profit from a prevention program.

Appetite For Life addressed this issue in different ways. Firstly, at the screening stage, all participants who showed no risk of developing an ED or only mild symptoms were given access only to the low intensity modules. All participants who were already suffering from severe symptoms were excluded from the program. Secondly, the program allowed for the intensity of support to be adjusted according to the needs and preferences of participants. As long as they did not develop severe symptoms, participants were able to use the different components of the program for as long and as intensively as they wanted. Those participants who developed severe symptoms during their participation were encouraged to use more intensive treatment components (consultation chat or referral to face-to-face counseling). 
Table 2. Acceptability and User Satisfaction

\begin{tabular}{|l|c|c|}
\hline \multicolumn{1}{|c|}{ Acceptability and user satisfaction } & N & (\% of participants) \\
\hline \hline Monitoring & & $(76,47 \%)$ \\
\hline good concept & $26 / 34$ & $(27,59 \%)$ \\
\hline feedback appropriate & $8 / 29,96 \%)$ \\
\hline helpful & $6 / 29$ & $(84,85 \%)$ \\
\hline Individual consultation chat & & $(17,39 \%)$ \\
\hline good concept & $28 / 33$ & \\
\hline helpful & $4 / 23$ & $(54,84 \%)$ \\
\hline Group consultation chat & & $(8,70 \%)$ \\
\hline good concept & $17 / 31$ & \\
\hline helpful & $2 / 23$ & \\
\hline Anonymous forum & & $(62,79 \%)$ \\
\hline good concept & $27 / 34$ & $(26,47 \%)$ \\
\hline helpful & $9 / 34$ & \\
\hline
\end{tabular}

Table 3. Comparison of Risk Groups: Acceptability and User Satisfaction

\begin{tabular}{|c|c|c|c|c|}
\hline Acceptability and user satisfaction & \multicolumn{2}{|c|}{ Moderate risk group } & \multicolumn{2}{|c|}{$\underline{\text { High risk group }}$} \\
\hline Yes & $6 / 8$ & $(75 \%)$ & $12 / 25$ & $(48 \%)$ \\
\hline No & $2 / 8$ & $(25 \%)$ & $13 / 25$ & $(52 \%)$ \\
\hline E-Mail from University & $1 / 8$ & $(12.50 \%)$ & $0 / 25$ & $(0 \%)$ \\
\hline Friends & $0 / 8$ & $(0 \%)$ & $1 / 25$ & $(4 \%)$ \\
\hline Internet research & $7 / 8$ & $(87.50 \%)$ & $15 / 25$ & $(60 \%)$ \\
\hline Other & $0 / 8$ & $(0 \%)$ & $9 / 25$ & $(36 \%)$ \\
\hline I had specific questions I wanted answered & $0 / 8$ & $(0 \%)$ & $3 / 25$ & $(12 \%)$ \\
\hline Curiosity & $3 / 8$ & $(37.50 \%)$ & $13 / 25$ & $(52 \%)$ \\
\hline Other & $2 / 8$ & $(25 \%)$ & $1 / 25$ & $(4 \%)$ \\
\hline \multicolumn{5}{|l|}{ Monitoring } \\
\hline good concept & $6 / 8$ & $(75 \%)$ & $19 / 25$ & $(76 \%)$ \\
\hline feedback appropriate & $4 / 7$ & $(57.14 \%)$ & $4 / 22$ & $(18.18 \%)$ \\
\hline helpful & $2 / 7$ & $(28.57 \%)$ & $4 / 22$ & $(18.18 \%)$ \\
\hline
\end{tabular}


Table 3. contd....

\begin{tabular}{|c|c|c|c|c|}
\hline Acceptability and user satisfaction & \multicolumn{2}{|c|}{ Moderate risk group } & \multicolumn{2}{|c|}{$\underline{\text { High risk group }}$} \\
\hline good concept & $6 / 7$ & $(85.71 \%)$ & $11 / 23$ & $(47.83 \%)$ \\
\hline helpful & $0 / 5$ & $(0 \%)$ & $2 / 18$ & $(11.11 \%)$ \\
\hline good concept & $7 / 8$ & $(87.50 \%)$ & $19 / 25$ & $(76 \%)$ \\
\hline helpful & $1 / 8$ & $(12.50 \%)$ & $8 / 25$ & $(32 \%)$ \\
\hline I have learned new things by participating in Appetite For Life & $1 / 8$ & $(12.50 \%)$ & $6 / 25$ & $(24 \%)$ \\
\hline Participation in Appetite For Life helped me to clarify certain questions & $1 / 8$ & $(12.50 \%)$ & $4 / 25$ & $(16 \%)$ \\
\hline I would use Appetite For Life again & $2 / 8$ & $(25 \%)$ & $11 / 25$ & $(44 \%)$ \\
\hline I would recommend Appetite For Life to a friend whose eating habits I worry about & $4 / 8$ & $(50 \%)$ & $12 / 25$ & $(48 \%)$ \\
\hline \multicolumn{5}{|l|}{ Is/ was participation in Appetite For Life helpful for you? } \\
\hline Yes, somewhat & $3 / 8$ & $(37.50 \%)$ & $12 / 25$ & $(48 \%)$ \\
\hline Neither helpful nor harmful & $4 / 8$ & $(50 \%)$ & $12 / 25$ & $(48 \%)$ \\
\hline No, it is harmful & $1 / 8$ & $(12.50 \%)$ & $1 / 25$ & $(4 \%)$ \\
\hline
\end{tabular}

Note. Participants were allowed to answer "don't know", if they had not used a specific component. Therefore, the total number of ratings per component varies between 5 and 8 in the "moderate risk" and between 18 and 25 in the "high risk" group.

The aim of targeting participants at-risk of developing an ED in the first instance was reflected in the actual sample of Appetite For Life users. Results show that Appetite For Life was particularly attractive for students who were at "high risk": i.e. $66 \%$ of all registered users fell within the "high risk" group.

Participants who registered in Appetite For Life were on average more impaired in terms of ED symptomatology than healthy norm samples, but less impaired compared to clinical norm samples. Registered participants were characterized by higher BMI values and scored significantly higher on the WCS and the BNTSI (SEED) compared to eligible participants who did not register on the program.

Offering an anonymous, low intensity program to prevent ED seems to have been well accepted by the target population. For about half of the participants Appetite For Life was the first occasion on which they had dealt with the subject ED. Taking into account the ED pathology of the participants, Appetite For Life served as an important first contact point for students with ED related impairment. This is in line with other studies investigating online ED programs, e.g. [24], and demonstrates that Appetite For Life helps to deliver support to students in need that might not otherwise seek face-to-face treatment or counseling. Easy access, anonymity, and a cost-free service are likely to be the main reasons why this internet-based service is so attractive to populations that may not have sought support on their own. Appetite For Life effectively uses these advantages to address underserved individuals in need.
The use of various Appetite For Life modules was relatively low, e.g. $16.67 \%$ of all active users didn't ever use the monitoring program, and $20.51 \%$ used it only once. Online treatment programs often report lower compliance and higher dropout rates compared with face-to-face interventions [25]. This might be due to higher anonymity and less perceived need for commitment. However, anonymous online programs might actually attract those individuals, who would have avoided engaging in a face-to-face intervention. Although the Appetite For Life acceptability questionnaire was completed by only $43.59 \%$ of all active users, one can assume that acceptability data was not systematically biased, since participants who completed the questionnaire did not differ from those who did not, in terms of level of impairment, BMI, age or WCS. However, our sample of acceptability data is very small and not sufficient to draw conclusive results.

In general, acceptability and satisfaction were rated more positively by "high risk" participants compared with "moderate risk" participants. Nevertheless, the acceptability of the program appears to have been relatively low. It was clear that individuals varied in terms of what they needed from the program and which components they actually used. Although the forum, the monitoring function, and the consultation chat were rated positively overall by roughly $80 \%$ of participants, each component was rated as being helpful by only about $20 \%$ of the users. As most of the participants did not utilize the consultation chats, it is obvious that this would have affected the degree to which they found it helpful. Nev- 
ertheless, participants indicated that they agreed with the concepts on which the program was based and it is therefore likely that participants would have utilized these components if needed.

The various modules were perceived differently by participants of the different risk groups. The monitoring function was rated as being 'appropriate' and 'helpful' by a higher percentage of the "moderate risk" participants compared with "high risk" participants, whereas the forum was rated as being helpful by a higher percentage of the "high risk" group. However, the number of participants, who used various components intensively enough to reliably evaluate their usefulness is relatively small. Further, we assume that differences in the appropriateness and helpfulness of the monitoring module are associated with the pre-defined feedback messages, which contain supportive feedback and preliminary self-help strategies. For "high risk" individuals, who may have addressed their ED symptoms/attitudes before participating in Appetite For Life, the feedback messages might not have contained much new information about selfhelp strategies, in comparison with those in the "moderate risk" group, who may have had less exposure to information and advice about EDs in the past. The group chat was rated as being a 'good concept' by most of the "moderate risk" participants, but only by about half of the "high risk" participants. We assume that some users, especially "high risk" participants, may have been avoidant of group settings due to feelings of fear and shame [6] preferring instead more personal ways of discussing their problems.

Although very little is known about compliance in emental health programs in general [25], we should take into account that there are no strict rules about utilization of the different components of Appetite For Life when evaluating the utilization of and compliance with the program. The main idea of individualizing support intensity implies that the use of a component should depend on the individual's level of impairment.

In general, "high risk" individuals tended to use the higher intensity modules (i.e., forum and monitoring) more intensively than "moderate risk" participants, whereas lowintensity modules (i.e. information pages) were used more by "moderate risk" participants than by "high risk" participants. This is consistent with the individualized strategy underlying the development of Appetite For Life.

\section{CONCLUSION}

Appetite For Life attracted students at-risk of developing an ED, which were relatively impaired compared to healthy samples. For most of these students, it was the first occasion on which they had accessed help, and we assume that most of them would not have used face-to-face counseling otherwise. Experiences with the program indicate that individualization of support can be implemented by providing Internetdelivered stepped-care modules that encourage the user to seek support according to his/her personal preferences and needs. However, the study showed that there is room for improvement concerning the compliance rates. A larger study is needed to more systematically address the questions of who uses the program and who benefits from it.

\section{REFERENCES}

[1] Keel PK, Dorer DJ, Franko DL, Jackson SC, Herzog DB. Postremission predictors of relapse in women with eating disorders. Am J Psychiatry 2005; 162: 2263-8.

[2] Strober M, Freeman R, Morrell W. The long-term course of severe anorexia nervosa in adolescents: Survival analysis of recovery, relapse, and outcome predictors over 10-15 years in a prospective study. Int J Eat Disord 1997; 22: 339-60.

[3] Berkman ND, Lohr KN, Bulik CM. Outcomes of eating disorders: A systematic review of the literature. Int J Eat Disord 2007; 40: 293-309.

[4] Steinhausen H. The outcome of anorexia nervosa in the 20th century. Am J Psychiatry 2002; 159: 1284-93.

[5] Steinhausen H, Weber S. The outcome of bulimia nervosa: Findings from one-quarter century of research. Am J Psychiatry 2009; 166: 1331-41.

[6] Stewart M, Keel PK, Schiavo RS. Stigmatization of anorexia nervosa. Int J Eat Disord 2006; 39: 320-5.

[7] Richard M, Bauer S, Kordy H. Relapse in anorexia and bulimia nervosa-A 2.5-year follow-up study. Eur Eat Disord Rev 2005; 13 : 180-90.

[8] Jacobi C, Hayward C, de Zwaan M, Kraemer HC, Agras WS. Coming to terms with risk factors for eating disorders: Application of risk terminology and suggestions for a general taxonomy. Psychol Bull 2004; 130: 19-65.

[9] O'Connell ME, Boat T, Warner KE. Preventing mental, emotional, and behavioral disorders among young people: Progress and possibilities. [Internet]. 2009 [cited 2010 May 10]; Available from: http://www.redi-bw.de/db/ebsco.php/search.ebscohost.com/login. aspx ?direct $=$ true $\& \mathrm{db}=$ psyh $\& A N=2009-10864-000 \&$ site $=$ ehost-live

[10] Taylor CB, Bryson S, Luce KH, et al. Prevention of eating disorders in at-risk college-age women. Arch Gen Psychiatry 2006; 63: 881-8.

[11] Newton MS, Ciliska D. Internet-based innovations for the prevention of eating disorders: A systematic review. Eat Disord 2006; 14 : 365-84.

[12] Haaga DAF. Introduction to the special section on stepped care models in psychotherapy. J Consult Clin Psychol 2000; 68: 547-8.

[13] Kordy H, Haug S, Percevic R. Patients differ-A plea for individually tailored service allocation. Eur Eat Disord Rev 2006; 14: 1-7.

[14] Bauer S, Moessner M, Wolf M, Haug S, Kordy H. ES[S]PRIT - An internet-based programme for the prevention and early intervention of eating disorders in college students. Br J Guid Couns 2009; 37 : 327-36.

[15] Stice E, Shaw H. Eating disorder prevention programs: A metaanalytic review. Psychol Bull 2004; 130: 206-227.

[16] Killen JD, Taylor CB, Hayward C, Wilson DM. Pursuit of thinness and onset of eating disorder symptoms in a community sample of adolescent girls: A three-year prospective analysis. Int J Eat Disord 1994; 16: 227-238.

[17] Bauer S, Winn S, Schmidt U, Kordy H. Construction, scoring and validation of the short evaluation of eating disorders (SEED). Eur Eat Disord Rev 2005; 13: 191-200.

[18] BZgA Essstörungen: Startseite [Internet]. [cited 2010 May 10]; Available from: http://www.bzga-essstoerungen.de/

[19] Percevic R, Lambert MJ, Kordy H. Computer-supported monitoring of patient treatment response. J Clin Psychol 2004; 60: 285-99.

[20] Reas DL, Williamson DA, Martin CK, Zucker NL. Duration of illness predicts outcome for bulimia nervosa: A long-term followup study. Int J Eat Disord 2000; 27: 428-34.

[21] Moessner M, Fassnacht D, Bauer S. Online assessment of eating disorders: The clinical and research inventory for eating disorders (CR-EAT). (in review).

[22] Fairburn CG, Bèglin SJ. Assessment of eating disorders: Interview or self-report questionnaire? Int J Eat Disord 1994; 16: 363-70. 
[23] Mond J, Hay P, Rodgers B, Owen C. Eating disorder examination questionnaire (EDE-Q): Norms for young adult women. Behav Res Ther 2006; 44: 53-62.

[24] Grunwald M, Wesemann D. Individual use of online-consulting for persons affected with eating disorders and their relatives-evaluation of an online consulting service. Eur Eat Disord Rev 2006; 14: 21825 .

[25] Melville KM, Casey LM, Kavanagh DJ. Dropout from Internetbased treatment for psychological disorders. Br J Clin Psychol [Internet]. 2009 Oct 1 [cited 2010 May 10]; Available from: http://www.ncbi.nlm.nih.gov/pubmed/19799804

Received: May 16, 2010

Revised: September 09, 2010

Accepted: September 14, 2010

(C) Lindenberg et al.; Licensee Bentham Open.

This is an open access article licensed under the terms of the Creative Commons Attribution Non-Commercial License (http://creativecommons.org/licenses/by-nc/3.0/) which permits unrestricted, non-commercial use, distribution and reproduction in any medium, provided the work is properly cited. 